\title{
Une perspective multimodale sur les pratiques d'hétéro-sélection du locuteur en classe
}

\section{Virginie Fasel Lauzon et Evelyne Pochon-Berger}

\section{(2) OpenEdition}

Journals

\section{Édition électronique}

URL : http://journals.openedition.org/pratiques/1625

DOI : 10.4000/pratiques. 1625

ISSN : 2425-2042

\section{Éditeur}

Centre de recherche sur les médiations (CREM)

\section{Édition imprimée}

Date de publication : 15 décembre 2010

Pagination : 105-130

\section{Référence électronique}

Virginie Fasel Lauzon et Evelyne Pochon-Berger, « Une perspective multimodale sur les pratiques d'hétéro-sélection du locuteur en classe », Pratiques [En ligne], 147-148| 2010, mis en ligne le 15 décembre 2010, consulté le 19 avril 2019. URL : http://journals.openedition.org/pratiques/1625; DOI : $10.4000 /$ pratiques. 1625 


\title{
Une perspective multimodale sur les pratiques d'hétéro-sélection du locuteur en classe
}

\author{
Virginie Fasel Lauzon, Evelyne Pochon-Berger
}

Université de Neuchâtel, Centre de linguistique appliquée

Les ${ }^{(1)}$ interactions en classe se caractérisent souvent par une asymétrie des rôles adoptés par les enseignants et les élèves. Cette asymétrie se reflète notamment dans la manière dont enseignants et élèves prennent la parole en classe. Par exemple, lever la main pour demander la parole est une pratique typiquement associée aux élèves. Inversement, la mention explicite du prochain locuteur (next speaker, Sacks et al., 1974) souhaité après une question est une pratique typiquement associée aux enseignants.

Les études sur les interactions en classe de langue, tout en soulignant la diversité des modalités de prise de parole en classe, montrent que la gestion de ces prises de parole, notamment la sélection du prochain locuteur, est très majoritairement effectuée par l'enseignant (van Lier, 1988 ; McHoul, 1978 ; Mehan, 1979 ; Mondada, 1995 ; Pekarek Doehler, 2002 ; Seedhouse, 2004 ; Sinclair \& Coulthard, 1975). Tout en s'inspirant de ces études, cet article se propose d'observer plus en détail les pratiques de sélection du prochain locuteur en classe de langue. Il se donne pour objectif de montrer que des microanalyses séquentielles et multimodales, c'est-à-dire centrées sur l'enchaînement des tours de parole ainsi que sur la coordination des regards, des gestes et des positionnements corporels des participants, permettent une compréhension fine de la manière dont la sélection du prochain locuteur est localement gérée en classe. Elles invitent à envisager l'accomplissement de la sélection comme le produit des ajustements conjoints de l'ensemble des participants, ajustements qui se déploient séquentiellement mais également de manière simultanée, plutôt que comme relevant de la responsabilité d'un seul participant, l'enseignant.

(1) Les auteures remercient Jean-François de Pietro ainsi qu'un relecteur anonyme pour leurs commentaires sur une première version de cet article. 


\section{L'organisation des prises de parole en classe de langue}

L'étude présentée dans cet article s'inscrit dans le cadre de l'analyse conversationnelle $(\mathrm{AC})^{(2)}$, dont l'un des travaux fondateurs (Sacks et al., 1974) propose une description de la «machinerie » des tours de parole dans des conversations ordinaires ( $c f$. aussi p. ex. Schegloff \& Sacks, 1973 ; Schegloff, 1968, 1981). Cette machinerie comporte deux composants organisationnels : un composant relatif à la construction des tours (turn-constructional component), c'est-à-dire à l'agencement interne de chaque tour, et un composant ayant trait à l'attribution des tours (turn-allocation component), c'est-à-dire à la transition d'un tour à l'autre et d'un locuteur à l'autre. Sacks et al. (1974) distinguent deux types de techniques relatives à l'attribution des tours de parole : des techniques d'auto-sélection - où un participant s'attribue le droit de prendre la parole - et des techniques d'hétéro-sélection - où un participant attribue à un autre le droit ou l'« obligation » de prendre la parole. La sélection du prochain locuteur s'organise dans les conversations ordinaires selon un ordre de préférence qui s'inscrit dans la logique séquentielle suivante :

1. Le locuteur en cours sélectionne un autre participant pour prendre la parole dès que son tour arrivera à sa fin.

2. Si aucun participant n'a été sélectionné par le locuteur en cours, alors un participant peut s'auto-sélectionner à la première occasion.

3. Si les deux possibilités précédentes n'ont pas été appliquées, alors le locuteur en cours poursuit son tour, et à la prochaine opportunité pour le transfert de la parole, les options 1 , puis 2 , s'appliquent à nouveau.

Comme dans les conversations ordinaires, les prises de parole en classe peuvent être auto- ou hétéro-initiées. Néanmoins, elles sont régies par des routines institutionnelles mises en place par les participants pour gérer les multiples contraintes propres aux interactions en milieu scolaire (Bange, 1992, 2006 ; Bigot, 1996 ; Cicurel, 2002), notamment : a) les interactions en classe impliquent généralement plus de trois participants ; b) les interactions en classe sont orientées vers un but, l'apprentissage ; c) les interactions en classe mettent en présence des participants dont les rôles sont asymétriques et complémentaires, généralement un enseignant et plusieurs élèves. Les routines institutionnelles mises en place par les participants et relatives à la prise de parole en classe servent à organiser les échanges de manière à éviter qu'une interaction n'éclate en plusieurs conversations parallèles ou que plusieurs participants ne prennent la parole simultanément, ce qui conduirait à un brouhaha général. Corrélativement, elles ont pour but d'instaurer un type d'interaction propice à l'apprentissage.

Les routines interactionnelles en classe impliquent des ajustements conjoints de l'enseignant et des élèves, adoptant des positionnements complémentaires. L'enseignant adopte le rôle de «maître de cérémonie » (Bouchard, 2005), planifiant des activités et instituant les règles de leur déroulement, dont font partie des règles relatives à la prise de parole des élèves. Ces règles, qui peuvent être explicitées par l'enseignant ou rester implicites, concernent l'ordre des prises de parole, la manière de demander la parole et de prendre la parole. Par exemple, l'enseignant

(2) Pour des introductions à l'AC, $c f$. p. ex. Gülich \& Mondada, 2001 ; ten Have, 1999 ; Markee, 2000 ; Schegloff, 2007. 
peut instituer un système de pré-attribution des tours (McHoul, 1978), demandant aux élèves de prendre la parole à tour de rôle, selon un ordre défini à l'avance (type « tour de table »). Il peut également hétéro-sélectionner des élèves sans qu'un ordre ait été défini à l'avance, de manière verbale (en nommant l'élève ou en utilisant un pronom à la deuxième personne) ou non-verbale (par exemple en pointant l'élève du doigt). Solliciter la parole en levant la main est généralement un comportement accepté voire encouragé par les enseignants. L'auto-sélection des élèves, c'est-à-dire la prise de parole en l'absence d'attribution explicite par l'enseignant, peut être un comportement accepté ou encouragé, mais également être un comportement dévalorisé voire sanctionné par l'enseignant, en fonction du type de classe (notamment de l'âge des élèves) ou d'activité.

Si l'instauration par les enseignants de règles pour la prise de parole en classe est bien connue, la manière dont les élèves s'orientent vers ces règles est documentée de manière encore peu détaillée. La présente contribution propose une description « micro » de l'hétéro-sélection d'élèves par l'enseignant. Elle montrera que la procédure de sélection doit être envisagée moins comme une démonstration du « contrôle » de l'enseignant sur l'interaction que comme le résultat des ajustements mutuels et négociés de l'ensemble des participants. Ces ajustements se déploient non seulement sur le plan séquentiel, mais également de manière simultanée, ce que seules des analyses multimodales (c.-à-d. prenant en compte le verbal et le non-verbal) permettent d'observer. Nos analyses présenteront deux phénomènes liés à la sélection du prochain locuteur par l'enseignant. En premier lieu, elles montreront que les élèves mettent en scène leur disponibilité ou leur indisponibilité à prendre la parole avant que l'enseignant ne procède à la sélection. Ces mises en scène sont prises en compte par l'enseignant au moment de la sélection. En second lieu, elles montreront que la négociation liée à la sélection du prochain locuteur peut également s'observer après que la sélection ait eu lieu : la sélection par l'enseignant d'un élève n'ayant pas mis en scène sa disponibilité à prendre la parole a des conséquences sur la suite de l'interaction et peut conduire à la sélection d'un autre locuteur.

\section{Données}

Nos analyses se basent sur un corpus de vingt périodes scolaires, filmées dans des classes de français langue seconde, auprès de deux enseignants (dix périodes par enseignant). Les enregistrements ont été effectués en Suisse alémanique au niveau du secondaire supérieur (dernière année de lycée, élèves âgés de 17-18 ans). Le dispositif d'enregistrement consiste en une caméra disposée face aux élèves, les enseignants n'apparaissant pas toujours dans le champ de vision de la caméra. Les enregistrements vidéo ont été transcrits intégralement en suivant les conventions de transcriptions habituelles en analyse conversationnelle ( $c f$. annexe).

Les activités enregistrées visent toutes, aux dires des enseignants, à la pratique de la communication orale et au développement de ressources relatives à la compétence d'interaction. Il s'agit de discussions portant sur un texte littéraire, un article de journal ou un thème de société ainsi que de jeux (jeux de rôles, devinettes, charades, création de récits, etc.), qui peuvent prendre place en classe entière, en demi-classes ou en petits groupes. 
Les enseignants explicitent la manière dont ils souhaitent que les élèves prennent la parole dans trois jeux (devinettes, création de récits, jeu du téléphone arabe), lors desquels ils mettent en place un système de pré-attribution des tours (chaque élève ou groupe d'élèves devant prendre la parole à tour de rôle, selon un ordre prédéfini). Dans les autres activités, les enseignants ne donnent pas de consignes sur la manière dont les élèves sont censés prendre la parole. Un survol d'autres données, enregistrées en classes de français langue première et seconde au secondaire inférieur et supérieur (corpus SPD, corpus CODI, corpus BâleWBS $)^{(3)}$, montre que les explicitations quant à la manière de prendre la parole sont fréquentes au secondaire inférieur (élèves âgés de 13-14 ans), où les enseignants demandent fréquemment aux élèves de lever la main et d'attendre que la parole leur soit attribuée, tandis qu'elles sont quasi-inexistantes au secondaire supérieur (nous n'avons trouvé qu'une seule occurrence pour un total de 54 périodes scolaires, dans laquelle l'enseignant demande aux élèves de s'auto-sélectionner: "je vous prie de ne pas vous annoncer de ne pas lever la main mais d'introduire spontanément votre opinion", SPD L2-secII-14).

Cette absence d'explicitation donne lieu à différentes manières de prendre la parole et de sélectionner le prochain locuteur dans les activités, à savoir auto-sélection, hétéro-sélection en réponse à un lever de main et hétéro-sélection en l'absence de lever de main. Dans certaines activités, une sorte de routine se met en place et une seule manière de sélectionner le prochain locuteur est observable. Dans d'autres, plusieurs manières de sélectionner le prochain locuteur peuvent coexister. Pour des raisons de cohérence et de place, les analyses qui suivent portent toutes sur des moments où l'enseignant procède à l'hétéro-sélection d'un élève après avoir posé une question.

\section{Mises en scène des disponibilités à répondre}

\subsection{Mettre en scène sa disponibilité à prendre la parole}

\subsubsection{Lever la main}

La mise en scène par les élèves de leur disponibilité à prendre la parole après une question de l'enseignant « à la cantonade » (Bouchard, 2005) s'observe premièrement par les gestes conventionnalisés que constituent les levers de doigts, de mains ou de bras. Les enseignants interprètent ces gestes comme des mises en scène par les élèves de leur disponibilité à répondre, voire comme des sollicitations des élèves pour répondre. Dans les interactions en classe entière, impliquant un grand nombre d'élèves que l'enseignant ne peut pas tous embrasser d'un seul regard, lever la main apparait comme le moyen le plus efficace pour attirer l'attention de l'enseignant et être sélectionné pour prendre la parole.

(3) Le corpus SPD comprend 22 périodes scolaires de français langue seconde enregistrées (audio) en 1993 au niveau du secondaire supérieur ( $c f$. Pekarek, 1999, pour une description détaillée) ; le corpus CODI comprend 44 périodes scolaires de français langue première et seconde enregistrées (audio + vidéo) au niveau des secondaires inférieur et supérieur (L1 secI : 12 périodes scolaires ; L1-secII, 12 périodes scolaires ; L2-secII, 20 périodes scolaires ; $c f$. Fasel Lauzon, 2009, pour une description détaillée) ; le corpus Bâle-WBS comprend 15 périodes scolairesde français langue seconde enregistrées (audio + vidéo) au niveau du secondaire inférieur. 
Le lever de main a déjà été documenté comme une pratique finement coordonnée avec le comportement de l'enseignant. Mondada (2009) montre par exemple la manière dont les élèves initient un lever de main à des moments opportuns dans le déroulement de l'interaction, à savoir au moment où l'enseignant ouvre une nouvelle séquence. La question de l'enseignant projette ainsi un « espace d'intervention » (Mondada, 2009 : 37) pour les élèves. Ces observations montrent l'analyse minutieuse que font les participants du déploiement séquentiel des conduites mutuelles. Dans nos données, nous observons en outre que la coordination des participants est visible à l'intérieur même de l'espace d'intervention ouvert par l'enseignant. L'exemple suivant révèle la subtile synchronisation du déploiement du geste avec le comportement de l'enseignant qui balaie la classe du regard à la recherche d'un volontaire pour répondre à la question :

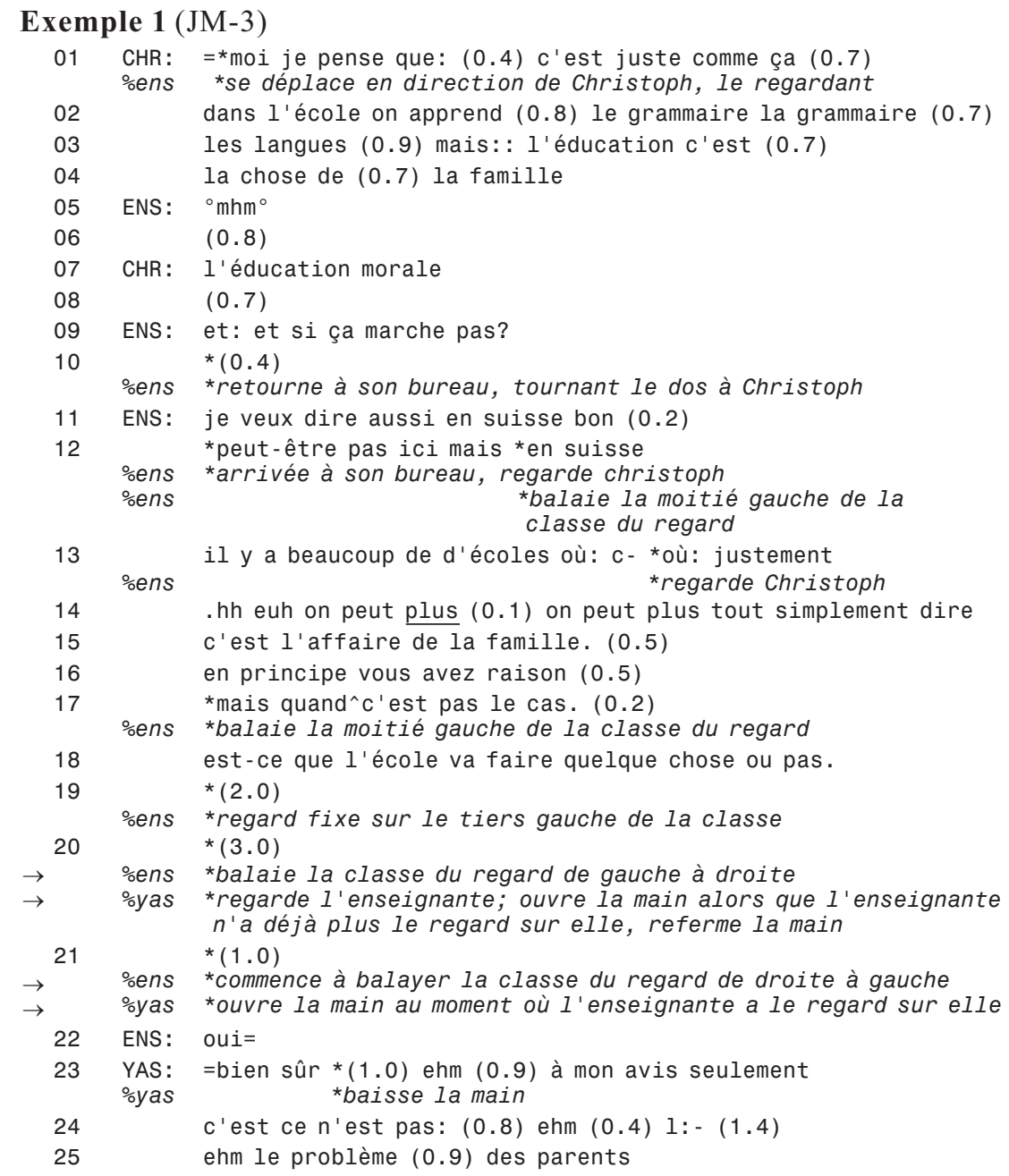

Au début de cet exemple, Christoph donne son opinion personnelle sur le rôle de l'école dans l'éducation des enfants (1.1-7). L'enseignante réagit à cette inter- 
vention au moyen d'une question qu'elle développe dans un long tour ("et si ça marche pas", 1.9). Durant son tour de parole, l'enseignante regarde alternativement Christoph $(1.12+13-16)$ et le reste de la classe, qu'elle balaie du regard (1.12 et 17). Cette alternance de regards permet à l'enseignante de présenter son tour à la fois comme une réaction adressée à un élève en particulier ( $c f$. aussi "en principe vous avez raison", 1.16) et comme une question ouverte à l'ensemble des élèves. A la fin de son tour, l'enseignante reformule sa question ("est-ce que l'école va faire quelque chose ou pas", 1.18) puis balaie la classe du regard à plusieurs reprises. Ce «balayage», qui prend au total six secondes, semble être pour l'enseignante un moyen de chercher un élève souhaitant prendre la parole (un willing next speaker, Mortensen, à paraitre) plutôt que de sélectionner arbitrairement un participant. Le balayage du regard manifeste ainsi un " adressage » à l'ensemble de la classe, au cours duquel se négocie une nouvelle distribution de la parole ( $c f$. Bouchard, 2005).

Après deux secondes de pause durant lesquelles son regard est dirigé sur le côté gauche de la classe, l'enseignante commence à déplacer son regard vers la droite. Dès le début du mouvement, Yasmine, assise au centre, lève le regard. Alors qu'elle s'apprête à croiser le regard de l'enseignante, Yasmine initie un mouvement pour lever la main. Cependant, au moment où sa main est levée, l'enseignante, qui a tourné la tête très vite, a déjà le regard dirigé trop à droite pour que Yasmine puisse être dans son champ de vision. Un contact visuel avec l'enseignante n'étant alors plus possible, Yasmine referme la main. Ces actions, qui se déroulent sur trois secondes, sont illustrées ci-dessous au moyen de captures d'écran :

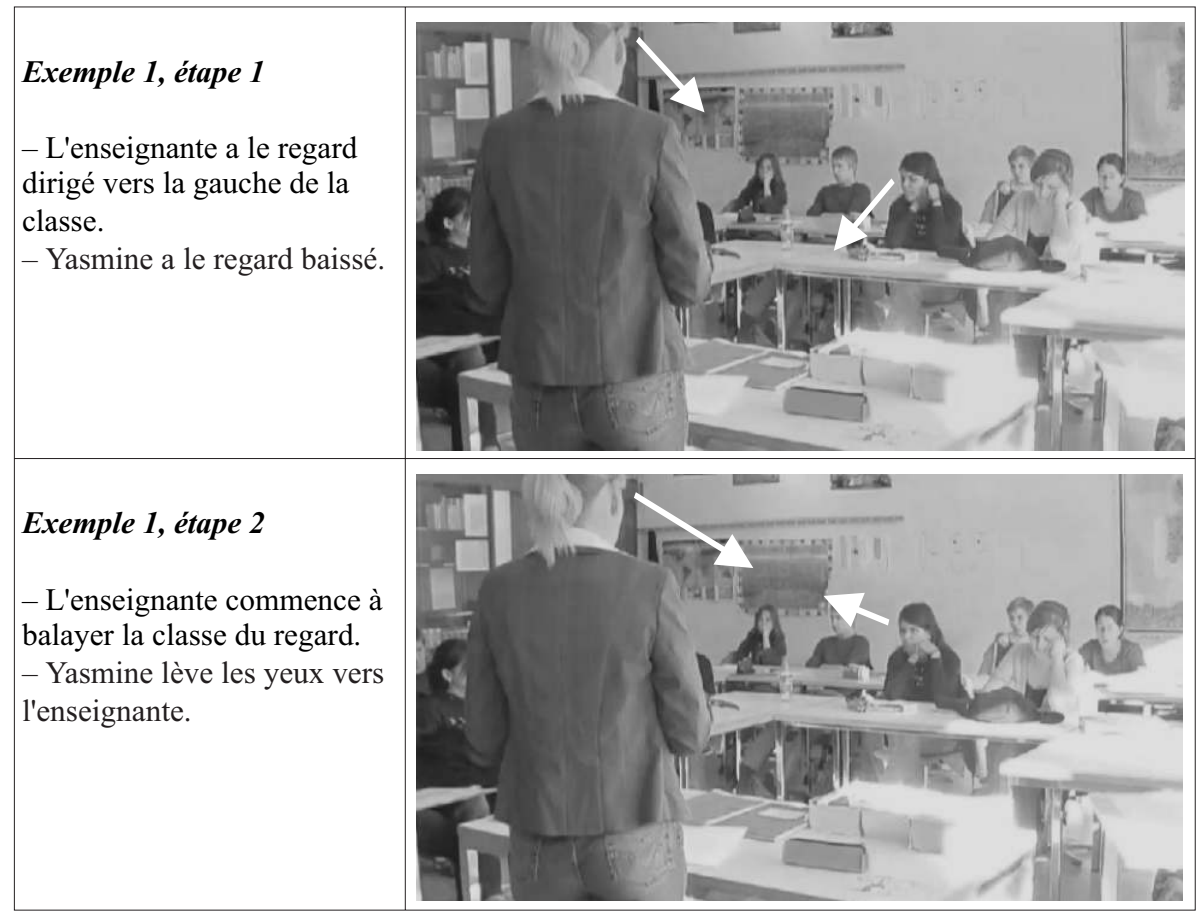




\section{Exemple 1, étape 3}

- L'enseignante « balaie » en passant par Yasmine.

- Yasmine lève la main.

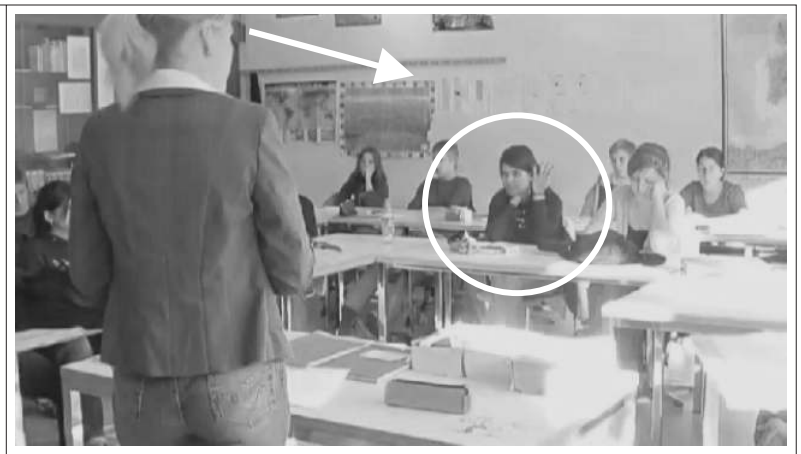

\section{Exemple 1, étape 4}

- L'enseignante termine son balayage vers la droite.

- Yasmine referme la main.

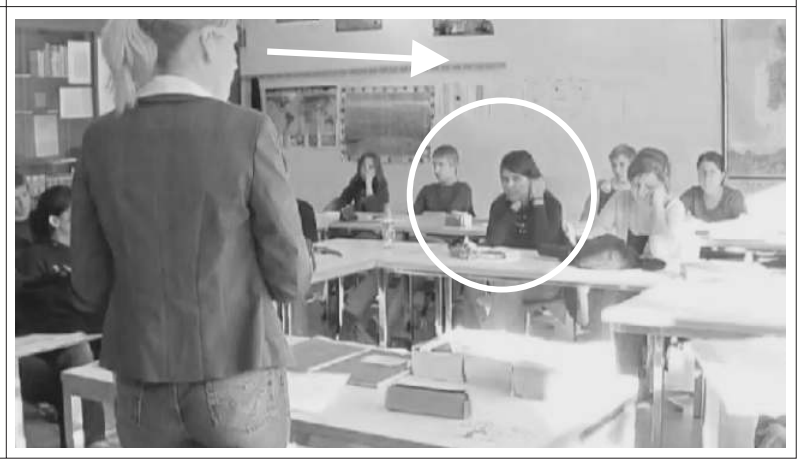

La main levée par l'élève et le balayage du regard par l'enseignante sont donc deux activités finement coordonnées. Néanmoins il semble qu'elles ne soient pas suffisamment synchrones : l'enseignante tournant la tête très vite, elle a déjà passé Yasmine lorsque celle-ci termine son geste de lever de main. Dans un second temps, ayant balayé la classe du regard sans trouver de candidat potentiel pour prendre le tour, l'enseignante recommence un balayage, cette fois de droite à gauche. Yasmine en profite pour lever à nouveau la main, en parfaite synchronisation avec le mouvement de balayage. Cette fois-ci, lorsque le regard de l'enseignante se pose sur Yasmine, cette dernière a déjà la main levée. L'enseignante lui accorde alors la parole (“oui”, 1.22) que l'élève prend aussitôt en baissant la main.

\section{Exemple 1, étape 5}

- L'enseignante recommence à balayer la classe du regard. - Yasmine relève la main. - L'enseignante arrête son regard sur Yasmine et lui accorde la parole.

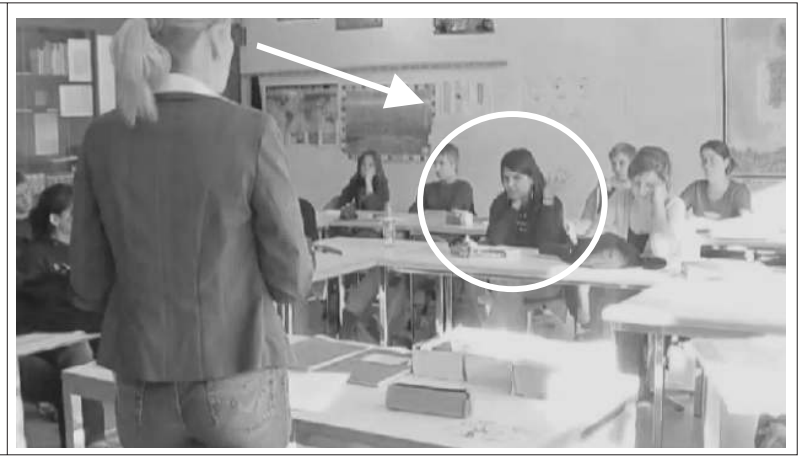

Le silence suivant la question de l'enseignante adressée à l'ensemble des élèves constitue un espace interactionnel ouvert à quiconque souhaite prendre la parole. En balayant l'ensemble du groupe du regard, de manière systématique, l'en- 
seignante met en scène sa recherche d'un locuteur pour répondre à sa question, permettant aux élèves de se faire reconnaître par l'enseignante comme disponibles pour répondre. Lever la main permet d'attirer l'attention de l'enseignante, pour autant que le geste soit synchronisé avec le déplacement de son regard. Ce déplacement continu rend la coordination des conduites mutuelles potentiellement difficile. Ainsi qu'on a pu l'observer, le premier lever de main de Yasmine a été « manqué » par l'enseignante.

Il apparaît ainsi que les levers de main des élèves ne sont pas simplement des pratiques conventionnalisées mettant en scène un état cognitif (la disponibilité, la disposition, la volonté à prendre la parole) mais des actions qui sont subtilement déployées (à la fraction de seconde près !) sur le plan interactionnel de manière à être synchronisées avec les regards de l'enseignant. Si la sélection proprement dite est accomplie par l'enseignant, elle est toutefois le résultat d'un processus finement coordonné entre enseignant et élève.

\subsubsection{Permettre l'établissement d'un contact visuel}

$\mathrm{Si}$, dans les activités en classe entière, lever la main est dans nos données le moyen le plus utilisé par les élèves pour mettre en scène leur disponibilité à répondre à la question de l'enseignant, c'est un moyen qui est peu utilisé dans les demi-classes. Il semble que lorsque l'enseignant a la possibilité d'embrasser quasiment la totalité des élèves d'un seul regard, ces derniers ont tendance à recourir à un moyen plus discret pour mettre en scène leur disponibilité à répondre : un bref regard jeté en direction de l'enseignant juste avant l'action de sélection. C'est ce qu'on observe dans cet exemple, prenant place dans une interaction en demi-classe :

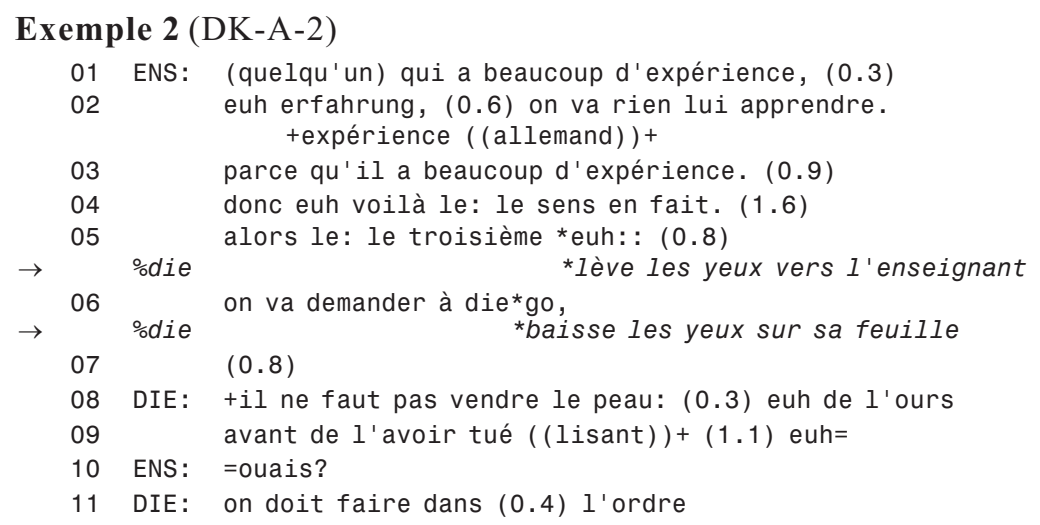

Cet exemple est tiré d'une activité dans laquelle les élèves ont reçu une liste de proverbes dont ils doivent tenter de trouver la signification. Au début de l'exemple, l'enseignant commente un proverbe qui vient d'être expliqué par un élève (« ce n'est pas à un vieux singe qu'on apprend à faire la grimace »; $c f .1 .1-3$ ). La séquence est close par l'enseignant au moyen d'un commentaire évaluatif ("donc euh voilà le : le sens en fait.", 1.4). L'enseignant initie alors une transition vers une nouvelle séquence ("alors le : le troisième", 1.5) avant de produire un marqueur de « remplissage » (“euh") suivi d'une pause (1.5) au moment où la sélec- 
tion d'un élève est attendue (dans les séquences précédant et suivant l'exemple, la sélection apparait également après la mention du numéro du proverbe : "le deuxième on va demander à andré", "le suivant on va demander à javier", etc.). Au moment de l'énonciation du marqueur, un élève, Diego, lève les yeux vers l'enseignant, alors que tous les autres ont le regard dirigé ailleurs (sur leur feuille de consigne, vers leurs mains, dans le vague). Cet élève est celui que l'enseignant sélectionne ("on va demander à diego", 1.6). Notons que cette sélection est ici effectuée par une nomination de l'élève. Cette procédure contraste avec les sélections d'élèves ayant levé la main, lesquelles sont généralement accomplies par un "oui" ( $c f$. exemple 1). Ces deux procédures traduisent ainsi une organisation séquentielle différente de la sélection du prochain locuteur : alors que lever la main est interprété par l'enseignant comme une sollicitation pour prendre la parole, une demande à laquelle le "oui" répond, l'échange de regards ne fait qu'indiquer une disponibilité de l'élève à répondre mais n'est pas interprété comme une sollicitation à proprement parler. La sélection dans ce cas est en apparence une initiative de l'enseignant.

\section{Exemple 2, étape 1}

- L'enseignant s'arrête juste avant la sélection.

- Diego tourne la tête dans la direction de l'enseignant (hors-champ, à l'autre bout de la table, sur la droite).

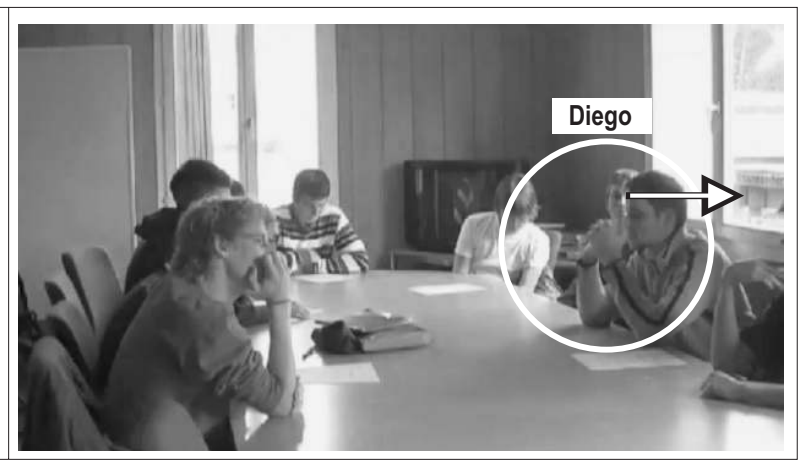

Aussitôt après que la sélection de Diego ait été rendue reconnaissable, c'est-àdire après l'énonciation de la première syllabe du prénom (aucun autre élève dans la classe n'a un nom dont la première syllabe est similaire à celle du prénom de l'élève, de sorte que l'énonciation de la première syllabe est suffisante pour que l'élève sache qu'il est sélectionné), ce dernier déplace son regard de l'enseignant à la liste de proverbes (1.6), pose la main sur la liste et lit le troisième proverbe (1.8-9), effectuant ainsi la tâche demandée.

\section{Exemple 2, étape 2}

- L'enseignant sélectionne Diego.

- Diego se met en « position de travail ».

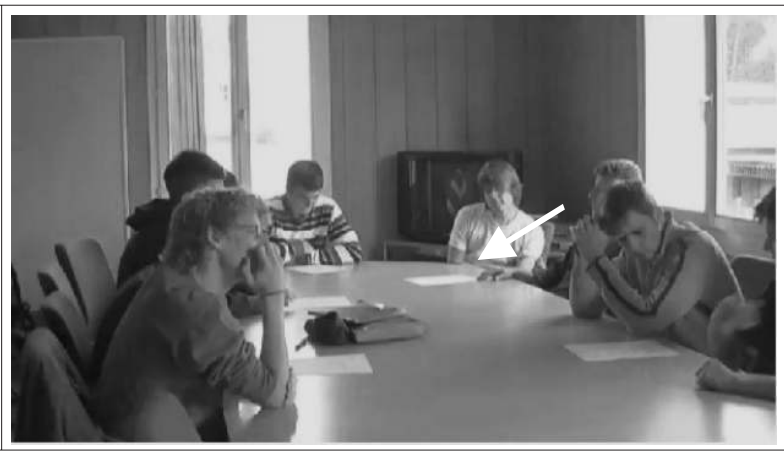


Cet exemple illustre une procédure récurrente dans les données et montre la manière dont regards et orientations corporelles mettent en scène des disponibilités à prendre la parole dans les interactions où le nombre d'élèves est peu élevé. En changeant la direction de son regard de manière synchronisée avec la transition, l'élève rend visible son attention (par opposition à « sa distraction » ou « son indifférence ») à l'activité en cours. Lorsque la direction du regard de l'élève permet la possibilité d'un contact visuel avec l'enseignant, cette possibilité est interprétée par l'enseignant comme la mise en scène d'une disponibilité à prendre la parole ( $c f$. Mortensen, à paraitre). Après la sélection de l'élève par l'enseignant, le changement de regard et de position de manière anticipée sur la fin du tour de l'enseignant incarne la « mise au travail » de l'élève, qui s'oriente dès ce moment-là vers la réalisation de l'activité attendue (lire un proverbe sur la feuille de consignes). Ce réarrangement corporel accomplit la transition entre la sélection et l'accomplissement de l'action projetée par la première partie de paire adjacente de l'enseignant. Ici comme dans la situation en classe entière où un élève lève la main ( $c f$. point 3.1.1), l'action de sélection doit donc s'envisager comme le résultat des ajustements mutuels et simultanés de l'enseignant et de l'élève.

\subsection{Ne pas mettre en scène sa disponibilité à prendre la parole}

Nous avons vu que les gestes et les regards des élèves peuvent leur permettre de mettre en scène leur disponibilité à prendre la parole. Inversement, certains comportements permettent aux élèves d'éviter de mettre en scène une telle disponibilité.

\subsubsection{Rester immobile}

Une manière pour les élèves de mettre en scène leur disponibilité à prendre la parole consiste à lever la main afin de se rendre potentiellement " remarquables » pour l'enseignant. Inversement, l'absence de geste et le maintien d'une certaine immobilité des positions corporelles après une question de l'enseignant permettent aux élèves de ne pas se rendre « remarquables » aux yeux de l'enseignant et d'éviter d'être sélectionnés, ce qu'illustre l'exemple suivant :

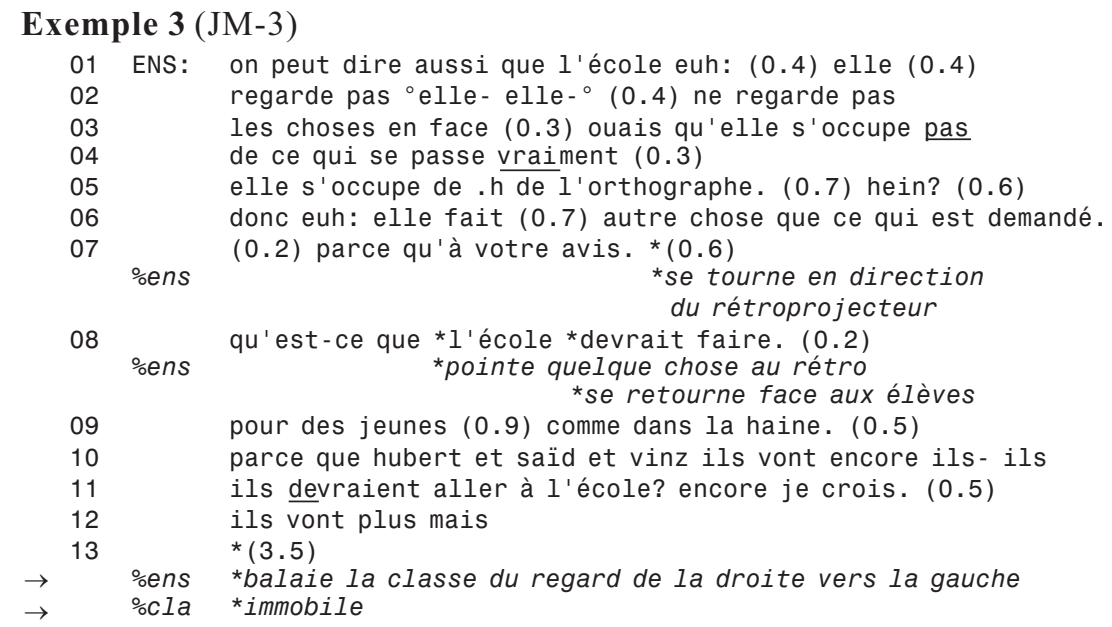




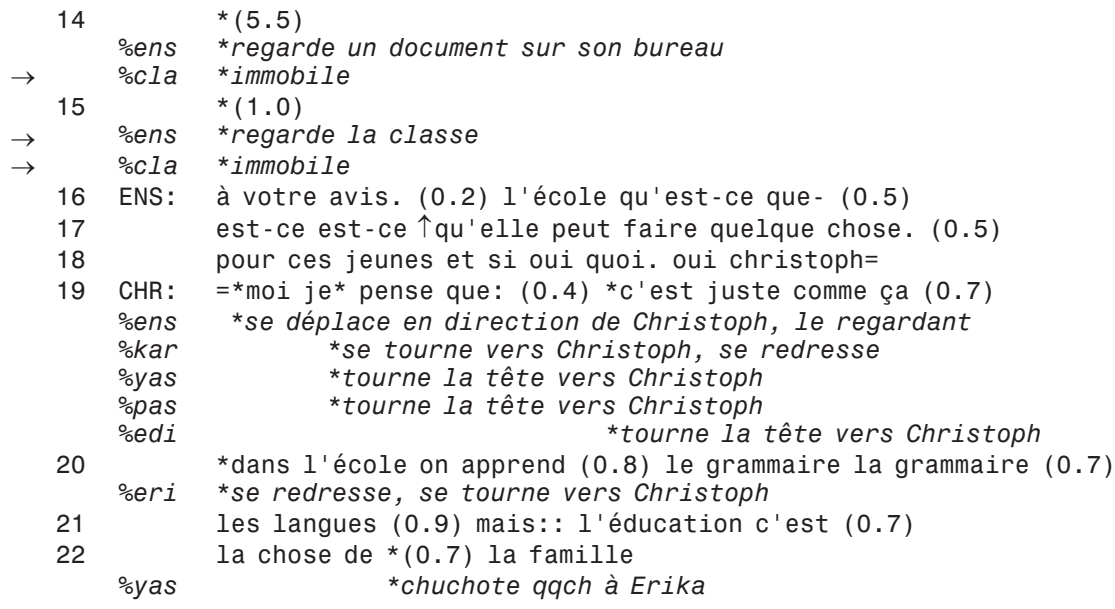

Cet exemple est tiré de la même activité que l'exemple 1, dans lequel une élève levait la main de manière synchronisée avec le déplacement du regard de l'enseignante. On observe également ici que l'enseignante pose une question adressée à l'ensemble des élèves (1.7-12), puis balaie la classe du regard à la recherche d'un volontaire (le balayage ne dure ici que 3.5 secondes, l'enseignante regardant ensuite un document se trouvant sur son bureau). Cependant, durant les dix secondes de silence qui suivent la question de l'enseignante, les élèves restent immobiles. Les deux captures d'écran ci-dessous, effectuées au début et à la fin de la pause, permettent d'observer cette immobilité :

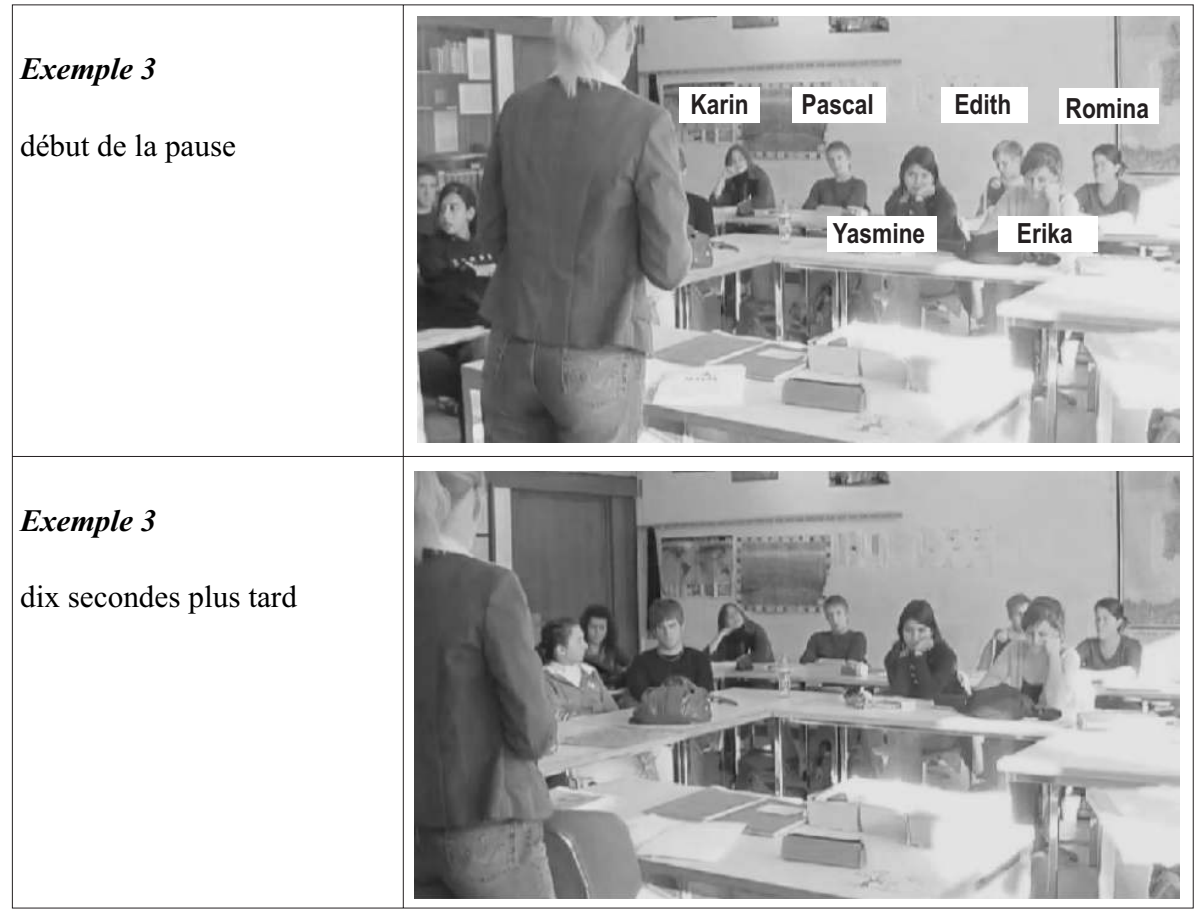


L'immobilité des élèves leur permet de ne pas se rendre « remarquables » sur le plan visuel. De plus, certains élèves adoptent une position corporelle peu adéquate pour prendre la parole ( $c f$. p. ex. position affaissée avec la tête appuyée sur la main de Karin). L'enseignante s'oriente vers les comportements des élèves comme traduisant l'absence de mise en scène de disponibilité pour prendre la parole. Plutôt que de sélectionner arbitrairement un élève, elle procède alors à une reformulation de sa question (1.16-18), qui fonctionne comme une relance. Il est intéressant d'observer que la reformulation ouvre de nouvelles possibilités de réponse : alors que la première formulation ("qu'est-ce que l'école devrait faire", 1.8 ) contient le présupposé que l'école « devrait faire » quelque chose, la seconde formulation ("l'école est-ce qu'elle peut faire quelque chose... et si oui quoi", 1.16-18) abandonne ce présupposé, offrant aux élèves la possibilité de présenter un point de vue selon lequel l'école ne « devrait pas » ou ne «peut pas » faire quelque chose. Suite à la deuxième formulation de la question, l'enseignante accorde la parole (1.18) à un élève qui lève probablement la main, comme en témoigne le “oui" précédant le nom de l'élève (qui n'est pas visible sur la vidéo). A noter que l'élève prenant la parole affirme que l'éducation est l'affaire de la famille plutôt que celle de l'école, ce qui peut être la raison pour laquelle cet élève n'a pas mis en scène sa disponibilité après la première version de la question, celle présupposant que l'école doit jouer un rôle dans l'éducation des enfants. Les élèves précédemment immobiles se remettent à bouger (changeant de position corporelle, tournant la tête, se parlant entre pairs, etc., $c f$. 1.19-21) dès le moment où Christoph est sélectionné. Cela suggère que leur immobilité avant la sélection traduisait bien une tentative de se rendre le moins « remarquables » possible et d'éviter ainsi d'être sélectionnés au moment où l'enseignante recherchait un locuteur potentiel : une fois la sélection d'un participant effectuée et le changement de locuteur accompli, les élèves peuvent recommencer à bouger sans risquer d'être sélectionnés.

\subsubsection{Rendre impossible le contact visuel}

Nous avons vu que dans les interactions en classe impliquant un petit nombre d'élèves, la mise en scène d'une disponibilité à prendre la parole est accomplie par une orientation du regard de l'élève en direction de l'enseignant, ce qui permet l'établissement d'un contact visuel. On observe également des cas où à l'inverse les élèves changent l'orientation de leur regard au moment où l'enseignant initie la phase de sélection de manière à rendre impossible un contact visuel entre eux et l'enseignant.

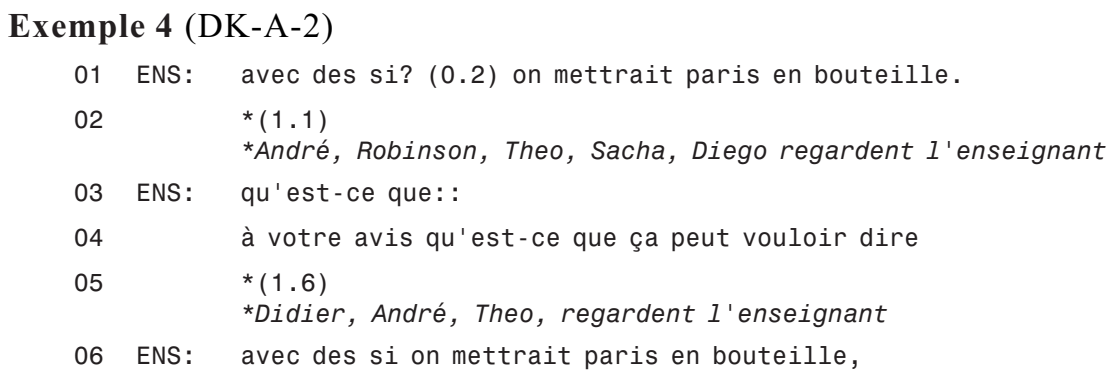




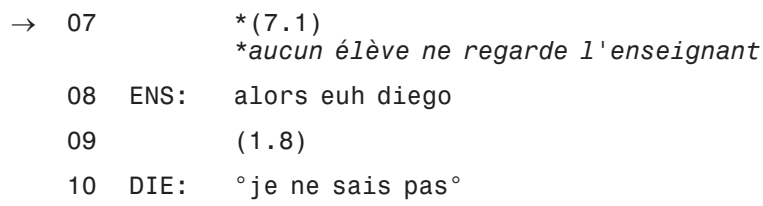

Cet exemple est tiré de la même activité que l'exemple 2, dans lequel un élève oriente son regard de manière à permettre un contact visuel avec l'enseignant, ce qui est interprété par ce dernier comme la mise en scène d'une disponibilité à répondre et conduit à une sélection de l'élève. Au début de l'exemple 4, l'enseignant énonce un proverbe et en demande la signification aux élèves (1.1-4; à noter que ce proverbe n'est pas inscrit sur la liste de proverbes que l'enseignant a distribuée aux élèves et que ces derniers ont rangée). Durant la pause qui suit l'énonciation du proverbe et précède l'énonciation de la question (1.2), cinq élèves ont le regard dirigé vers l'enseignant. Après l'énonciation de la question (1.4), deux élèves détournent le regard, deux maintiennent leur regard et un nouvel élève s'oriente vers l'enseignant. Cependant, alors que l'enseignant répète le proverbe avec une intonation continuative, ce qui projette la procédure de sélection comme imminente, la totalité des élèves détourne le regard. Les changements de regard peuvent être observés dans ces trois captures d'écran :

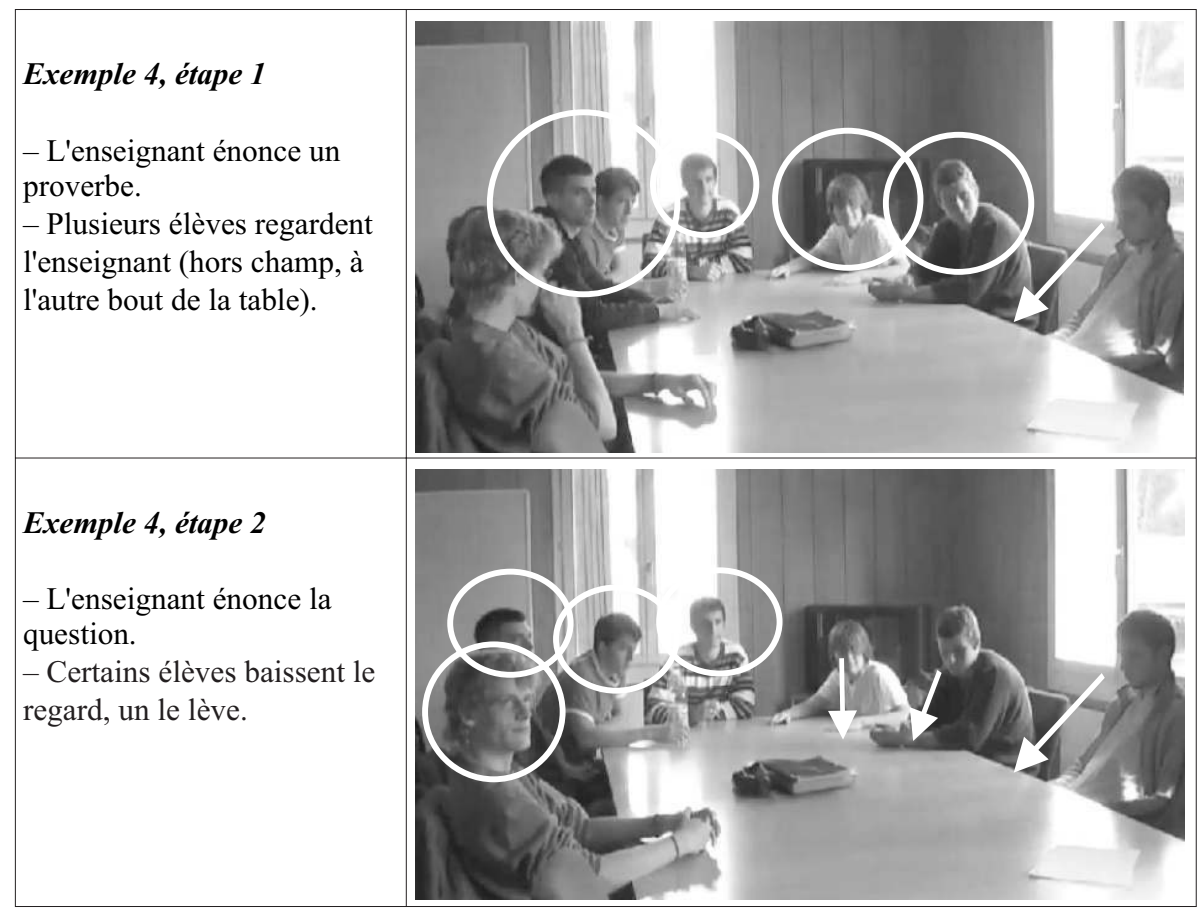




\section{Exemple 4, étape 3}

- L'enseignant répète le proverbe avec intonation continuative.

- Les élèves détournent le regard, rendant impossible le contact visuel.

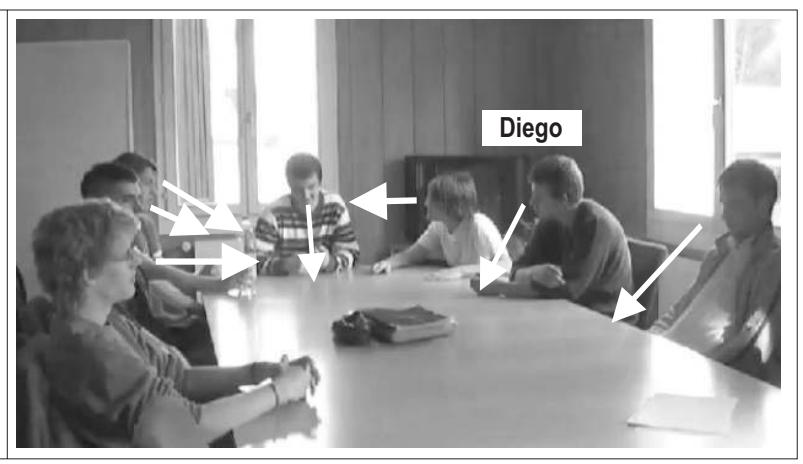

Ainsi, alors que les élèves dirigent leur regard vers l'enseignant à un moment où il n'y a pas de pertinence pour un changement de locuteur (c'est-à-dire avant que la première partie de paire adjacente - la demande d'explication - ne soit formulée), ils détournent le regard au moment pertinent pour la sélection du prochain locuteur (à la fin de la question), rendant le contact visuel avec l'enseignant impossible. Cette modification de la direction des regards incarne l'évitement d'un comportement (permettre l'établissement d'un contact visuel) qui pourrait être interprété par l'enseignant comme un indice de disponibilité à prendre la parole ( $c f$. aussi Mortensen, à paraitre). Après un long silence de 7.1 secondes, et alors qu'il n'a pas pu entrer en contact visuel avec un des élèves, l'enseignant sélectionne néanmoins l'un d'entre eux, Diego (1.6). Enfin, l'élève se déclare incapable de répondre à la question (1.8), ce qui suggère que le retrait de son regard incarnait bien une absence de disponibilité (ou de capacité) à répondre à la question de l'enseignant.

En résumé, les participants s'orientent vers la mise en scène d'une disponibilité à prendre la parole, ou au contraire vers l'absence d'une telle mise en scène (voire vers la mise en scène d'une indisponibilité), au moment précis où la sélection du prochain locuteur devient pertinente dans le déroulement séquentiel des activités. La disponibilité à répondre est manifestée par les élèves au travers de comportements non-verbaux : en levant la main (en classe entière) ou en dirigeant leur regard vers l'enseignant de manière à permettre l'établissement d'un contact visuel (en demi-classe). Si les élèves disposent de ressources pour mettre en scène leur disponibilité, ils exploitent ces mêmes ressources de manière inversée pour éviter de la mettre en scène : l'immobilité des élèves contraste avec le mouvement de lever de main et de même, le détournement du regard contraste avec la direction du regard vers l'enseignant. L'enseignant s'oriente vers ces indices non-verbaux au moment d'attribuer la parole en sélectionnant quand cela est possible un élève qui s'est montré disponible pour répondre. Ces observations montrent ainsi que l'hétéro-sélection des élèves par l'enseignant doit souvent s'envisager moins comme résultant d'une décision prise de manière individuelle et imposée par l'enseignant aux élèves que comme résultant d'un accomplissement conjoint et négocié auquel les élèves participent activement.

Ces exemples montrent en outre la manière dont cet ajustement des conduites mutuelles se fonde sur une analyse en temps réel que font les participants des détails de l'interaction. D'un côté, les élèves anticipent les cours d'action (p. ex. anticipation de la direction d'un regard ou d'une sélection imminente dans la 
progression du tour de l'enseignant) de façon à pouvoir rendre compte de leur disponibilité ou indisponibilité à prendre la parole. De la même manière, les comportements non-verbaux (contact visuel, lever de main, immobilité) produits par les élèves à des moments séquentiels spécifiques sont interprétés à leur tour par l'enseignant comme des indices de leur disponibilité ou indisponibilité. Cette disponibilité ou indisponibilité est ainsi "mise en scène" par les élèves, c'est-à-dire rendue publique - et dès lors reconnaissable pour la personne allant effectuer la sélection à proprement parler - par des comportements non-verbaux produits à des moments opportuns dans le déroulement de l'activité.

\section{Sélections d'élèves en l'absence de mises en scène de disponibilité}

\subsection{Sélectionner un élève « remarquable »}

Les mises en scène par les élèves de leurs disponibilités à prendre la parole jouent un rôle déterminant dans la procédure de sélection du prochain locuteur par les enseignants : comme nous l'avons montré, les enseignants tendent à sélectionner des élèves qui rendent reconnaissable leur disponibilité à prendre la parole. Inversement, les enseignants semblent éviter, lorsque cela est possible, de sélectionner des élèves qui ne se montrent pas disponibles, préférant reformuler leurs questions plutôt que de procéder à une sélection aléatoire ( $c f$. ex. 3), de manière à encourager la participation des élèves à la procédure de sélection. Cependant, il existe des situations dans lesquelles aucun élève ne manifeste de disponibilité à répondre, et dans lesquelles la question de l'enseignant peut difficilement être reformulée de manière différente ( $c f$. ex. 4). En l'absence d'indices de disponibilité, sur quelles bases l'enseignant procède-t-il à la sélection ? Il semble que dans certains cas, la sélection soit aléatoire ${ }^{(4)}$, comme dans l'exemple 4. Cet exemple apparait après l'explication de onze autres proverbes. Diego n'ayant jusqu'ici expliqué que le troisième proverbe et la demi-classe ne comptant que neuf élèves, il est possible que l'enseignant le sélectionne parce qu'il n'a pas pris la parole depuis longtemps. Mais d'autres élèves également ont été peu interrogés, deux élèves n'ayant d'ailleurs pas encore été interrogés une seule fois, ce qui renforce l'idée selon laquelle la sélection de Diego par l'enseignant relèverait d'une procédure arbitraire ${ }^{(5)}$.

La sélection arbitraire apparait cependant rarement dans nos données. Au contraire, il semble que lorsque cela est possible, les enseignants aient tendance à interroger l'élève dont le comportement est « remarquable » car différent de ce-

(4) Les «techniques » pour sélectionner les élèves, par exemple en choisissant alternativement un élève à droite et à gauche, peuvent également être considérées comme des sélections aléatoires. Nous rappelons que notre article ne porte que sur des activités dans lesquelles l'ordre de sélection des élèves n'est pas déterminé à l'avance, au contraire d'activités de type « tour de table» par exemple, dans lesquelles il est explicité au moment de la consigne que chaque participant prendra la parole dans un ordre défini.

(5) Il est également possible que certains enseignants aient tendance à interroger toujours le même élève (la «bonne poire ») quand aucun élève ne met en scène sa disponibilité. Cela ne semble cependant pas être le cas dans nos données. 
lui de ses pairs, même si ce caractère remarquable n'est pas dî̀ à la mise en scène d'une disponibilité à répondre (comme le lever de main ou l'orientation du regard dirigée vers l'enseignant). Les exemples suivants illustrent cette situation :
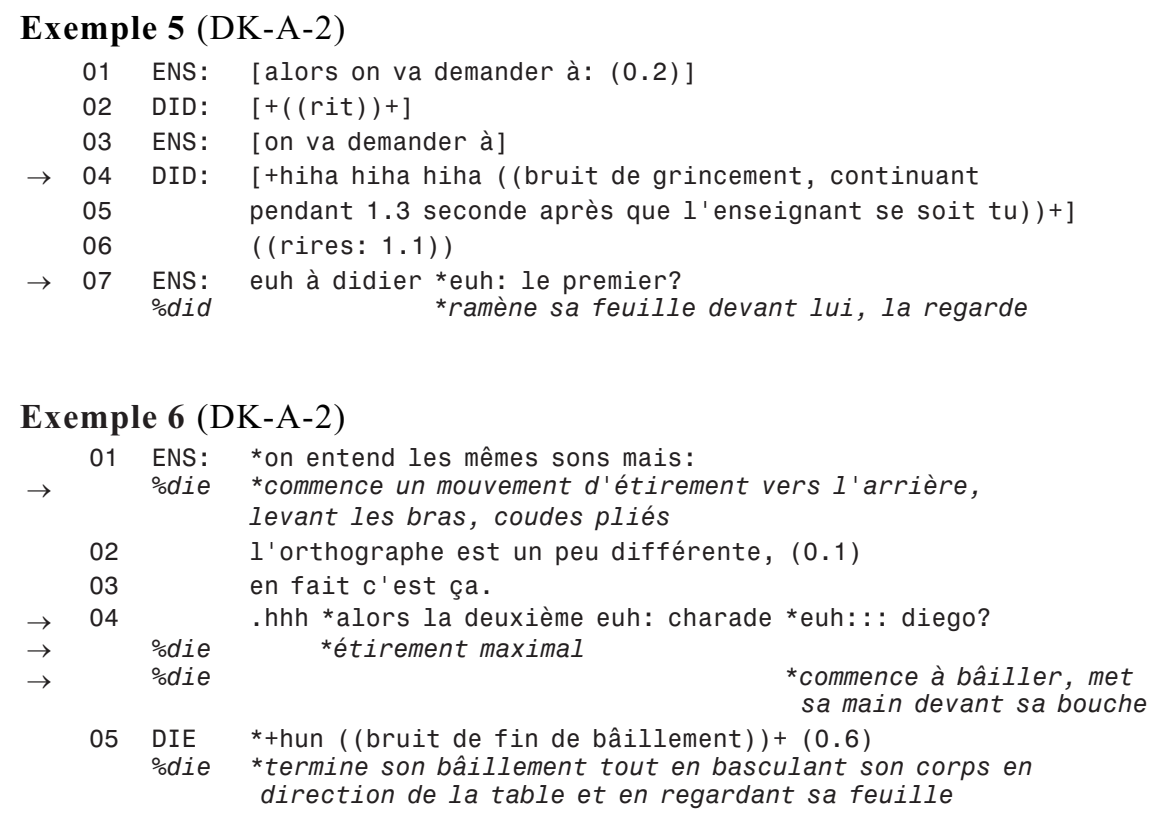

L'exemple 5 est tiré de la même activité que les exemples 2 et 4 . L'exemple débute immédiatement après une phase de l'activité dans laquelle les élèves ont eu pour tâche de trouver la signification de proverbes par groupes de deux. Durant cette phase, les élèves se sont mis à discuter en suisse-allemand d'un sujet sans rapport avec l'activité. Au début de l'extrait, l'enseignant prend la parole pour annoncer et introduire la nouvelle phase de l'activité ("alors on va demander à", 1.1). Au moment où l'enseignant prend la parole et initie la procédure de sélection, aucun élève ne le regarde. Dans cette perspective, la répétition de l'annonce de sélection après une auto-interruption ("on va demander à", 1.3) peut être un moyen pour l'enseignant de gagner du temps dans l'espoir d'entrer en contact visuel avec un élève qui mettrait ainsi en scène sa disponibilité à répondre. Aucun élève ne regarde cependant l'enseignant, ni lorsqu'il commence son tour, ni lorsqu'il répète l'annonce de sélection. Au contraire, les élèves ont tous le regard dirigé vers un de leur pair, Didier, qui est en train de parler au moment où l'enseignant prend la parole (1.2). L'élève poursuit son tour en chevauchement avec celui de l'enseignant, terminant par un bruit qui fait rires ses camarades (1.4-6). Aucun élève ne se montre donc disponible pour être sélectionné par l'enseignant, l'attention des élèves étant maintenue sur la conversation en suisse-allemand initiée pendant la phase de travail en groupes. Quand les rires cessent, l'enseignant reprend la parole et poursuit son projet communicatif ("à", 1.7). Il choisit Didier pour prendre la parole. A ce moment-là, Didier est l'élève le plus « remarquable» sur le plan visuel et verbal : il est le dernier des élèves à avoir pris la parole et il a l'attention de tous ses camarades, qui ont le regard dirigé vers lui. 


\section{Exemple 5}

- Didier parle et fait un bruit. - Les autres élèves regardent Didier et rient après le bruit. - L'enseignant sélectionne Didier.

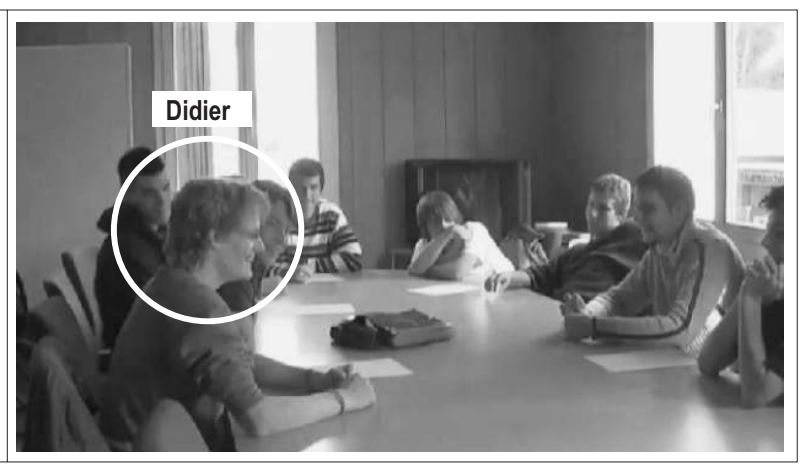

Dans l'exemple 6, tiré d'une activité sur des charades, aucun élève ne regarde l'enseignant au moment où la sélection devient imminente ("alors la deuxième euh charade euh:::", 1.4). A nouveau, l'enseignant doit donc procéder à la sélection d'un élève en l'absence de mise en scène de disponibilité. A ce moment-là, les élèves sont tous en «position de travail », les avant-bras posés sur la table et le regard sur leur feuille de consigne, sauf Diego, qui est appuyé sur son dossier, en train de s'étirer les mains derrière la tête. C'est cet élève que l'enseignant décide de sélectionner. Ainsi, dans ces deux exemples, l'enseignant sélectionne l'élève dont le comportement est le plus « remarquable» sur le plan verbal et/ou non-verbal, ce qui apparait de manière récurrente dans nos données.

\section{Exemple 6}

- Les élèves sont en

« position de travail ».

- Diego s'étire.

- L'enseignant sélectionne Diego.

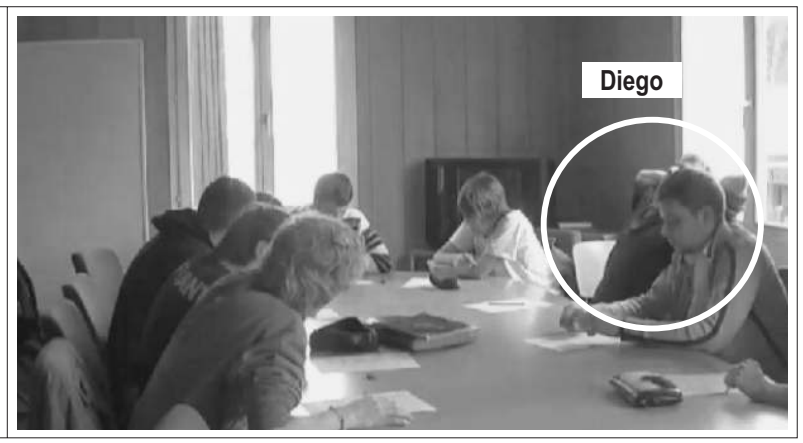

Dans ces deux exemples, l'élève sélectionné est le plus « remarquable » mais apparait également comme peu concentré sur l'activité. Dans l'exemple 5, Didier semble en partie responsable du fait qu'aucun autre élève ne se présente comme disponible pour prendre la parole : en parlant, il attire l'attention de ses camarades et les détourne de l'activité en cours. L'enseignant interrompt cette activité parallèle par la sélection de son «meneur ». Dans l'exemple 6, si Diego ne dérange pas ses camarades, il adopte une position qui peut traduire fatigue ou ennui et n'est pas adéquate pour travailler (elle ne lui permet pas de voir sa feuille de consignes). La sélection de Diego par l'enseignant peut donc être un moyen de « remettre à l'ordre » un élève et de le réengager dans l'activité. 


\subsection{Conséquences de la sélection en l'absence de mise en scène de disponibilité}

Lorsqu'un enseignant sélectionne un élève qui a mis en scène sa disponibilité à prendre la parole, la suite de la séquence se déroule sans problème particulier : l'élève prend la parole, immédiatement ou après une pause, et propose une réponse à la question de l'enseignant. Dans quelques rares cas, l'élève qui prend la parole après avoir levé la main n'accomplit pas une deuxième partie de paire adjacente mais ouvre une séquence insérée (insertion sequence, Schegloff, 2007) pour poser une «question sur la question ». Néanmoins on n'observe jamais, après la sélection d'un élève ayant mis en scène sa disponibilité, une réticence de l'élève à prendre la parole ou une déclaration d'incapacité à répondre à la question. En revanche, lorsque l'élève sélectionné n'a pas mis en scène sa disponibilité à répondre, la suite de l'interaction peut prendre différentes tournures. Dans la plupart des cas, la sélection est suivie de l'adoption par l'élève sélectionné d'une « position de travail », s'il n'était pas déjà dans cette position au moment où il a été sélectionné. Cela s'observe notamment dans les exemples 5 et 6 , où l'élève sélectionné se rapproche de la table et dirige son regard vers sa feuille de consigne avant de prendre la parole (ex. $5,1.7$; ex. 6, 1.5). Après ce moment de transition entre la sélection de l'élève et sa prise de parole, plusieurs options sont possibles. Une première possibilité consiste pour l'élève à accomplir la deuxième partie de paire demandée par l'enseignant. C'est ce que fait Didier dans l'exemple suivant, qui présente la suite de l'exemple 5 :

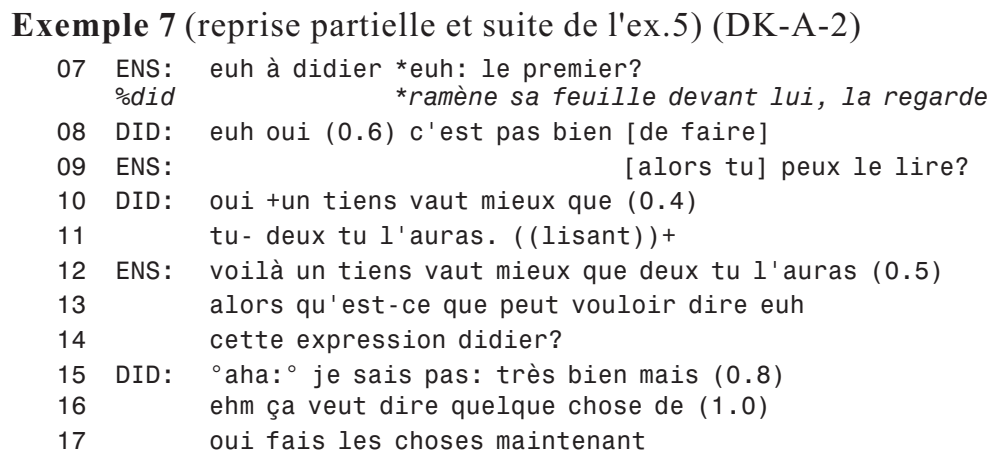

Dès qu'il entend son prénom, Didier ramène sa feuille devant lui. Il commence ensuite son tour en répondant positivement à l'invitation de l'enseignant ("euh oui", 1.8) ; au contraire, lorsque l'élève sélectionné a mis en scène sa disponibilité, le tour commence immédiatement par la réponse de l'élève, sans ratification de la sélection ( $c f$. ex. $1,1.23$, ex. $2,1.8$ ). Son orientation vers l'espace de travail et la ratification verbale de la sélection contribue ici à la transition entre la discussion entre élèves et la réponse à l'enseignant. De plus, contrairement à ce qui se passe dans l'exemple 2 (1.8-9), tiré de la même activité, l'élève ne commence pas ici par lire le proverbe à haute voix, ce qui a été demandé par l'enseignant dans une consigne présentée antérieurement. L'absence de lecture du proverbe conduit à une interruption de l'enseignant ("tu peux le lire", 1.9) et à un « recommencement » de la tâche par l'élève. La sélection d'un élève n'ayant pas mis en 
scène sa disponibilité a donc des conséquences observables sur la manière dont l'activité est initiée, la transition entre la demande de l'enseignant et la réaction de l'élève étant plus fluide lorsque l'élève sélectionné a mis en scène sa disponibilité.

Quoique la manière dont l'élève s'engage dans l'activité soit peu fluide, et quoique Didier annonce n'être pas certain de sa réponse ("je sais pas très bien", 1.15), l'activité poursuit son cours sans problème majeur. Ce n'est cependant pas toujours le cas : il arrive fréquemment qu'un élève sélectionné par l'enseignant en l'absence de mise en scène de disponibilité se déclare incapable de répondre à la question qui lui est posée ( $c f$. "je ne sais pas", ex. 4, 1.8). De telles déclarations d'incapacité doivent être envisagées comme des tentatives mises en place par les élèves pour éviter d'accomplir la seconde partie de paire adjacente malgré la sélection, plutôt que comme le reflet de leur état cognitif. Dans certains cas une affirmation d'incapacité peut être traitée comme peu crédible par les participants eux-mêmes, comme dans l'exemple suivant :

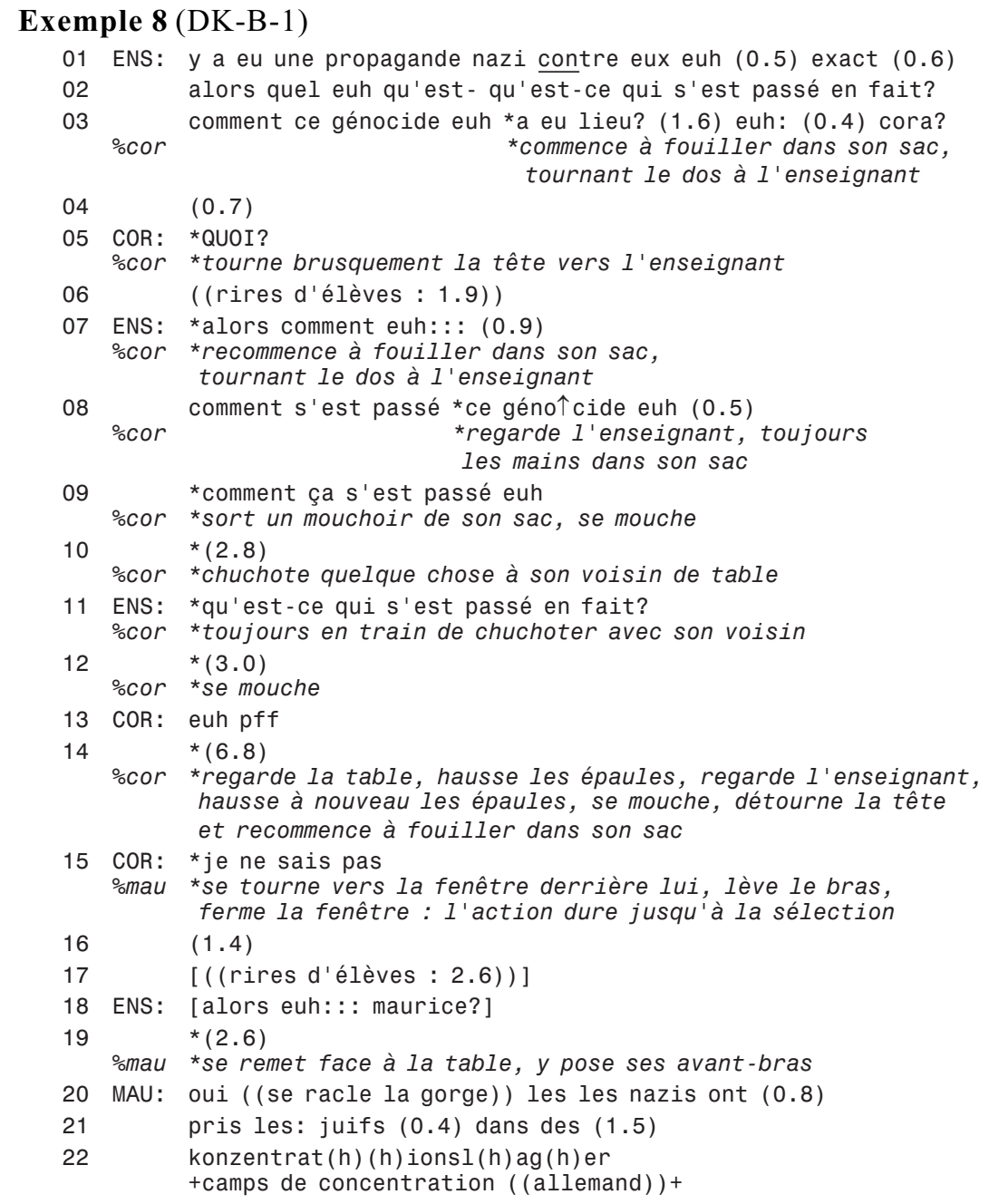


Au début de cet exemple, l'enseignant demande aux élèves de lui dire comment s'est passé le génocide nazi (1.1-3). Vers la fin de sa question, Cora commence à fouiller dans son sac, le dos tourné à l'enseignant. Après une pause, l'enseignant sélectionne cette élève au comportement " remarquable » car différent de celui des autres élèves, et traduisant potentiellement un manque d'attention pour l'activité en cours (1.3). Contrairement à ce qu'on a vu dans les autres exemples, Cora ne se remet pas en position de travail aussitôt qu'elle s'entend sélectionnée par l'enseignant. Presque une seconde se passe sans réaction de sa part (1.4) avant qu'elle ne se retourne, non pour proposer une réponse à la question mais pour ouvrir une séquence de réparation (“QUOI ?", 1.5) qui semble considérée peu appropriée de la part de ses pairs, comme en témoignent leurs rires (1.6). L'enseignant procède alors à plusieurs répétitions de sa question (1.7-11) en réaction à la demande de réparation (other-initiated repair, Schegloff et al. 1977). L'élève, plutôt que d'adopter une position d'écoute, recommence à chercher dans son sac, en sort un mouchoir, se mouche, puis se met à chuchoter avec son voisin de table. Ces comportements mettent en scène une forme d'indisponibilité de l'élève (le besoin de se moucher passant avant la mise en scène de l'écoute) mais aussi de réticence à se positionner comme réceptrice de la question et prochaine locutrice. La forte expiration ("pff", 1.13), les haussements d'épaules et le retrait du regard (1.14) contribuent également à mettre en scène cette réticence.

Après une longue pause (1.14), Cora affirme qu'elle ne peut pas répondre à la question ("je ne sais pas", 1.15). Il est peu probable qu'une élève arrivée à la fin du lycée ignore tout du génocide nazi. La réaction des élèves, qui se mettent à rire (1.17), témoigne d'une orientation vers le manque de crédibilité de l'affirmation de Cora. Celle-ci apparait plutôt comme un moyen pour Cora de ne pas répondre à la question de l'enseignant de façon socialement acceptable car justifiée (on ne peut pas répondre si on ne sait pas), et ce alors même que son comportement durant toute la séquence met en scène une indisponibilité et une réticence prononcée à prendre la parole. L'affirmation permet effectivement à l'élève de se retirer de l'activité, l'enseignant procédant alors à la sélection d'un autre élève (“alors euh:: maurice", 1.18 ; à noter qu'à nouveau, l'élève sélectionné est le plus « remarquable » sur le plan de son comportement non-verbal puisqu'il est en train de fermer la fenêtre). L'affirmation d'incapacité à répondre aura donc permis à Cora de cesser d'être « l'élève sélectionnée » au profit (ou au détriment) d'un de ses camarades.

Dans la majorité des cas, l'affirmation d'incapacité à répondre d'un élève est suivie d'un changement de « répondant» (par hétéro- ou auto-sélection d'un autre élève). Cependant, dans de plus rares cas, l'enseignant insiste pour que ce soit l'élève sélectionné qui réponde, au moins de manière minimale. C'est ce qui se ce produit dans l'exemple suivant, qui apparait à la suite de l'exemple 6, où l'enseignant avait sélectionné un élève en train de s'étirer :

\footnotetext{
Exemple 9 (reprise partielle et suite de 6) (DK-A-2)

04 ENS:

\%die

odie

.hhh *alors la deuxième euh: charade *euh: : : diego?

*étirement maximal

*commence à bâiller, met sa main devant sa bouche
} 


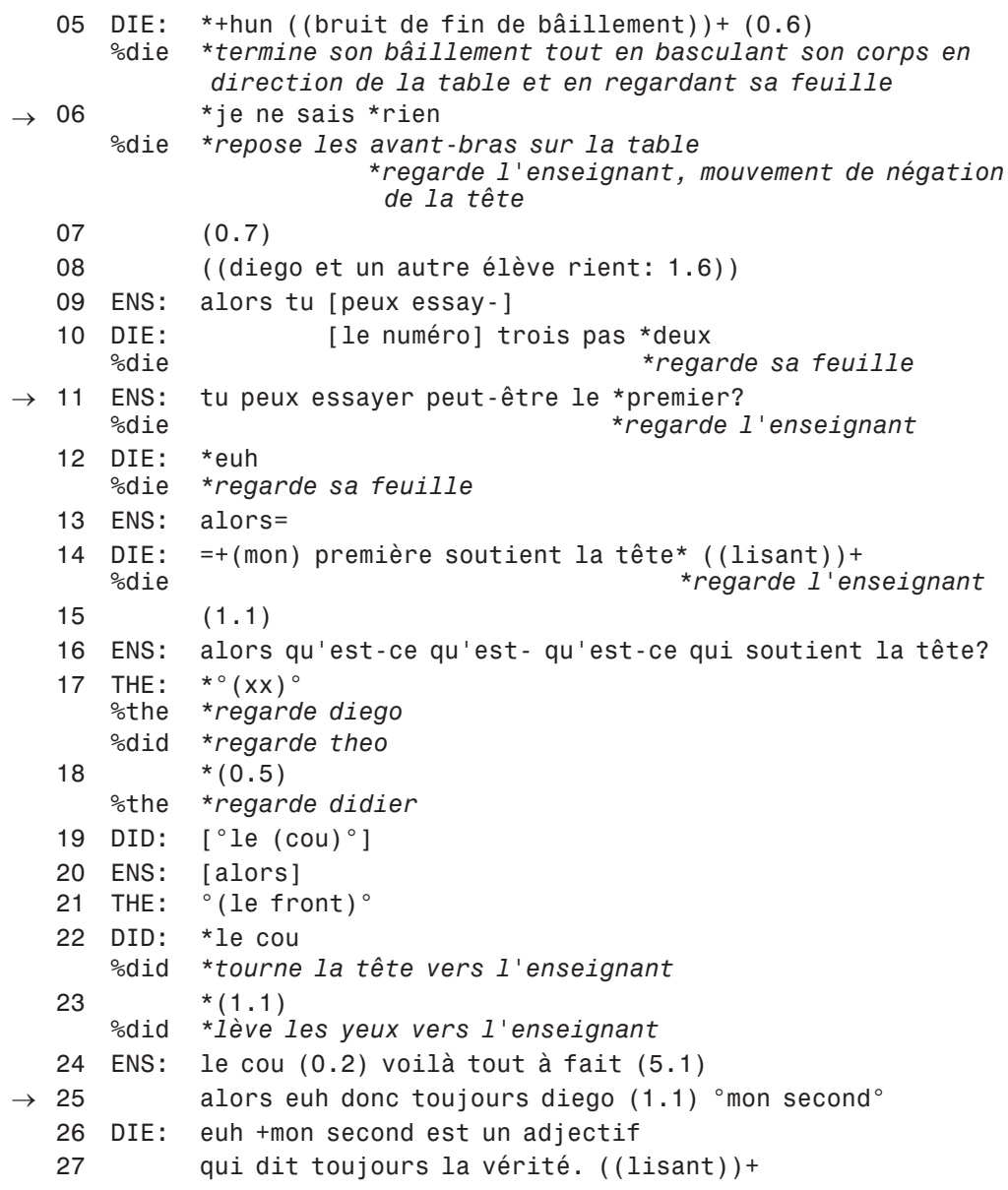

Après avoir été sélectionné, Diego adopte une « position de travail » (1.5-6) mais affirme son incapacité à répondre à la question de l'enseignant ("je ne sais rien", 1.6). Alors qu'il demande à effectuer la troisième charade plutôt que la deuxième (1.10), l'enseignant insiste et demande à Diego d'essayer de trouver le premier mot de la charade (1.11 et 13). Diego s'engage alors dans la tâche en lisant le premier segment de la charade (1.14), mais ne propose pas de solution, poursuivant sa mise en scène d'une incapacité à effectuer la tâche ( $c f$. la pause, 1.15). Après une relance de l'enseignant ("qu'est-ce qui soutient la tête", 1.16), on assiste à l'engagement de Theo et Didier dans la tâche (1.17-22) qui proposent des réponses en se regardant mutuellement ("le cou", 1.19, "le front", 1.21). L'un d'eux, Didier, adresse ensuite sa proposition à l'enseignant en s'auto-sélectionnant ("'le cou", 1.22). On observe donc ici un changement dans les modalités de participation et de sélection des élèves.

L'enseignant évalue positivement la réponse de Didier ("voilà tout à fait", 1.24), avant de revenir à Diego, lui demandant de poursuivre l'activité ("donc toujours diego mon second", 1.25). En acceptant la réponse de Didier, l'enseignant valide implicitement l'auto-sélection de l'élève, ce qui peut encourager d'autres élèves à s'engager activement dans l'activité même sans avoir été hété- 
ro-sélectionnés. En maintenant Diego engagé dans l'activité, quoiqu'il ait affirmé ne pas avoir trouvé les réponses de la charade, l'enseignant s'assure que Diego reste attentif à l'interaction, alors que cet élève, par son bâillement et son étirement, avait l'air plutôt distrait. Cet extrait montre donc une manière intéressante dont l'enseignant peut gérer les conséquences découlant de la sélection d'un élève n'ayant pas mis en scène sa disponibilité et affirmant son incapacité à répondre, ce qui lui permet de maintenir l'engagement de l'élève dans l'activité tout en encourageant la participation active d'autres élèves.

En résumé, lorsqu'un enseignant doit sélectionner un élève en l'absence de mise en scène de disponibilité, il tend à sélectionner l'élève dont le comportement est le plus « remarquable » (dans le sens où il se démarque de celui de ses pairs), souvent parce qu'il a l'air peu attentif, ou à défaut un autre élève, de manière arbitraire. La sélection d'un élève n'ayant pas mis en scène préalablement sa disponibilité peut être problématique et a des conséquences observables sur la suite de l'interaction. Si, dans certains cas, l'élève sélectionné accomplit l'action demandée, la transition vers celle-ci est dans tous les cas moins fluide. Par ailleurs, de manière récurrente, les élèves sélectionnés affirment ne pas être capables de répondre à la question, ce qui leur permet parfois de se retirer de l'activité : l'enseignant tend alors à sélectionner un autre élève pour répondre. Déclarer son incapacité est également une manière pour les élèves de produire une action conforme et socialement acceptée (une justification) face à ce qui a été projeté antérieurement, c'est-à-dire de réagir à une hétéro-sélection de l'enseignant, sans toutefois fournir la réponse attendue. Dans certains cas, les enseignants insistent sur la participation de l'élève sélectionné. Cette insistance permet de maintenir l'élève engagé dans l'activité tout en encourageant d'autres élèves à y participer activement, ce qui semble particulièrement intéressant en tant que moyen pour augmenter le nombre de participants engagés dans une activité et pour diversifier les opportunités de participation en classe de langue.

\section{Conclusion}

Cet article s'est centré sur l'hétéro-sélection d'élèves pour répondre à des questions énoncées par des enseignants. Il s'est attaché à montrer que 1'accomplissement de la sélection du prochain locuteur est le résultat des ajustements mutuels des participants. Les gestes et les échanges de regards jouent un rôle clé dans le processus d'attribution de la parole en ce qu'ils permettent de mettre en scène les disponibilités des élèves. Les mises en scène de disponibilité ou l'absence de telles mises en scène sont prises en compte par les enseignants au moment de la sélection. De manière plus générale, il apparait que les enseignants tendent à sélectionner l'élève dont le comportement est le plus « remarquable», autrement dit l'élève qui se comporte différemment de ses pairs, que ce soit parce qu'il lève la main ou regarde l'enseignant, mettant en scène sa disponibilité, ou au contraire parce qu'il a l'air dissipé ou inattentif. Dans le premier cas, la sélection témoigne d'une interprétation par l'enseignant de l'élève comme étant disponible, et d'une réaction face à cette disponibilité. Dans le second cas, se produisant lorsqu'aucun élève ne s'est présenté comme disponible, la sélection permet à l'enseignant de (ré)intégrer dans l'activité l'élève qui semble peu attentif. La 
sélection d'un élève n'ayant pas mis en scène sa disponibilité, voire dont le comportement semble témoigner d'une « indisponibilité », a des conséquences sur la suite de l'interaction : la transition entre la sélection et la réponse de l'élève est moins fluide, et il arrive que l'élève sélectionné affirme être incapable de répondre à la question. Dans ce dernier cas, deux réactions de l'enseignant sont observables : l'enseignant sélectionne un autre élève pour répondre, ou au contraire insiste pour que l'élève sélectionné tente de répondre lui-même, ce qui permet de maintenir l'élève engagé dans l'activité tout en donnant à d'autres élèves l'opportunité de prendre la parole, notamment en s'auto-sélectionnant.

Les analyses séquentielles et multimodales présentées dans cet article ont permis de documenter les processus de coordination des participants. Elles ont montré que la prise en compte des gestes, des regards et des positionnements corporels dans l'analyse s'avère essentielle pour comprendre l'organisation des interactions en classe de langue, puisque les participants exploitent ces éléments non-verbaux comme des ressources pour se coordonner, s'ajuster, construire le sens de leurs conduites réciproques ( $c f$. Goodwin, 2000 ; Mondada, 2004). Les ajustements entre les participants sont donc observables non seulement sur le plan séquentiel ( $c f$. p. ex. l'adoption d'une « position de travail » après la sélection) mais également de manière simultanée ( $c f$. p. ex. les mises en scène de disponibilité simultanées à la mise en scène de la recherche d'un prochain locuteur par l'enseignant). Ces observations invitent à repenser la manière d'envisager l'hétéro-sélection en classe de langue comme le résultat d'une procédure de négociation non-verbale et d'un travail d'interprétation des conduites mutuelles prenant place entre l'ensemble des participants. Elles soulèvent des interrogations quasi-philosophiques sur la question du « libre choix » dans la participation à une interaction sociale. Sur un plan davantage appliqué, nos analyses pourraient être utilisées dans le cadre de la formation des enseignants pour être confrontées aux représentations que les enseignants se font de la manière dont ils sélectionnent leurs élèves, afin d'ouvrir la discussion sur les procédures de sélection en classe, les échanges non-verbaux, ainsi que sur la gestion des conséquences d'une sélection (par exemple, d'un élève réagissant en déclarant être incapable de répondre). 


\section{Références}

BANGE, P. (1992) : «A propos de la communication et de l'apprentissage en L2 (notamment dans ses formes institutionnelles) », AILE 1, 53-85.

Bigot, V. (1996) : «Converser en classe de langue : mythe ou réalité ?», Les Carnets du Cediscor 4, 33-46.

BOUCHARD, R. (2005) : «Les interactions pédagogiques comme polylogues », LIDIL 31, 139-155.

CiCUREL, F. (2002) : «La classe de langue. Un lieu ordinaire, une interaction complexe », AILE 16, 145-163.

FASEL LAUZON, V. (2009) : L'explication dans les interactions en classe de langue : organisation des séquences, mobilisation de ressources, opportunités d'apprentissage. Thèse de doctorat : Université de Neuchâtel (disponible en ligne sur http ://doc.rero.ch/lm.php ?url=1000,40,4,20090909095645HR/Th_FaselV.pdf).

GoodwIN, C. (2000) : «Action and embodiment within situate human interaction », Journal of Pragmatics 32, 1489-1522.

GÜliCH, E. \& MONDADA, L. (2001) : «Konversationsanalyse. Analyse conversationnelle », in G. Holtus, M. Metzeltin \& Ch. Schmitt (éds.), Lexikon der Romanistischen Linguistik. Vol. I. Tübingen : Max Niemeyer Verlag, 196-250.

MARKEE, N. (2000) : Conversation Analysis. Mahwah : Lawrence Erlbaum.

MCHOUL, A. (1978) : «The organization of turns at formal talk in the classroom », Language in Society 7, 183-213.

MeHAN, H. (1979) : Learning Lessons. Social Organization in the Classroom. Cambridge : Harvard University Press.

MONDADA, L. (2004) : «Temporalité, séquentialité et multimodalité au fondement de l'organisation de l'interaction : le pointage comme pratique de prise de tour ", Cahiers de Linguistique Française 26, 269-292.

— (2009) : «La production de l'intelligibilité de l'action : une approche multimodale des procédés de selection des locuteurs dans les interactions en classe ", Revue de didactologie des langues-cultures et de lexiculturologie $153,25-40$.

MORTENSEN, K. (à paraitre) : « Selecting Next-Speaker in the Second Language Classroom : How to Find a Willing Next-speaker in Planned Activities », Journal of Applied Linguistics.

PEKAREK, S. (1999) : Leçons de conversation. Dynamiques de l'interaction et acquisition de compétences discursives en classe de langue seconde. Fribourg : Editions Universitaires.

PEKAREK DOEHLER, S. (2002) : «Formes d'interaction et complexité des tâches discursives dans des activités conversationnelles en classe de L2 », in $\mathrm{Ci}$ curel, F. \& Véronique, D. (éds.), Discours, action et appropriation des langues. Paris : Publications de la Sorbonne Nouvelle. 117-130.

SCHEGLOFF, E. A. (1968) : «Sequencing in Conversational Openings », American Anthropologist 70(6), 1075-1095. 
- (1981): «Discourse as an Interactional Achievement : Some Uses of "uh huh" and Other Things that Come Between Sentences », in D. Tannen (éd.), Analyzing Discourse : Text and Talk. Washington D.C.: Georgetown University Press, 71-93.

- (2007) : Sequence Organization in Interaction. A Primer in Conversation Analysis, vol. 1. Cambridge : Cambridge University Press.

Schegloff, E. A., Jefferson, G. \& SACKS, H. (1977) : « The Preference for Self-Correction in the Organization of Repair in Conversation », Language 53(2), 361-382.

Schegloff, E. A. \& SACKS, H. (1973) : «Opening up Closings », Semiotica VIII(4), 289-327.

Seedhouse, P. (2004) : The Interactional Architecture of the Language Classroom : A Conversation Analytic Perspective. Malden : Blackwell.

Sinclair, J. McH. \& Coulthard, R. M. (1975) : Towards an Analysis of Discourse. The English used by teachers and pupils. London : Oxford University Press.

TEN Have, P. (1999) : Doing Conversation Analysis. A Practical Guide. London : Sage.

VAN LIER, L. (1988) : The Classroom and the Language Learner. London : Longman. 


\section{Annexe \\ Conventions de transcription}

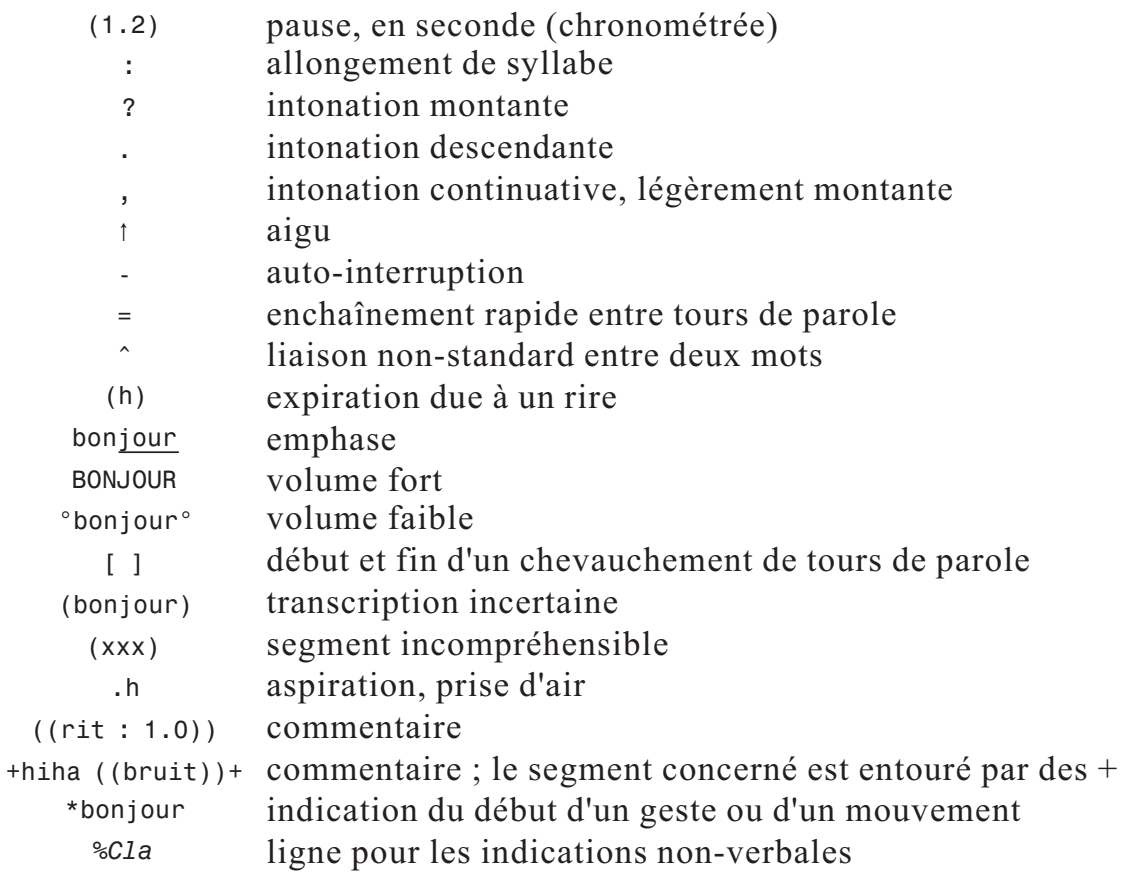

